\title{
A brain-lesion pattern based algorithm for the diagnosis of posttraumatic olfactory loss*
}

\author{
Jörn Lötsch',2, Nicole Reither ${ }^{3}$, Vasyl Bogdanov³, Antje Hähner ${ }^{3}$, \\ Alfred Ultsch ${ }^{4}$, Konstanze Hill5, Thomas Hummel ${ }^{3}$ \\ 'Institute of Clinical Pharmacology, Goethe - University, Frankfurt am Main, Germany \\ ${ }^{2}$ Fraunhofer Project Group Translational Medicine and Pharmacology (IME-TMP), Frankfurt am Main, Germany \\ ${ }^{3}$ Smell \& Taste Clinic, Department of Otorhinolaryngology, TU Dresden, Dresden, Germany \\ zDataBionics Research Group, University of Marburg, Marburg, Germany \\ ${ }^{5}$ Department of Neuroradiology, TU Dresden, Dresden, Germany
}

Rhinology 53: 365-370, 2015

DOI:10.4193/Rhino15.010

*Received for publication:

January 20, 2015

Accepted: April 9, 2015

\begin{abstract}
Background: Brain areas processing olfactory information exhibit functionally relevant morphological dynamics. This suggests the exploitation of anatomical information in the diagnosis of an olfactory dysfunction. Following previous identifications of olfactory eloquent areas such as the olfactory bulbs and tracts, we focused at a brain-morphology based algorithm for establishing the diagnosis of olfactory loss following brain injury.
\end{abstract}

Methodology: Forty-one patients with a history of head trauma dated back $40 \pm 39$ months, and additional 23 patients without head trauma, were assessed for damages in 11 olfaction-relevant brain areas using magnetic resonance imaging (MRI). Olfactory function was derived from the use of a standardized, reliable and validated olfactory test. An olfactory diagnostic algorithm was derived following classification and regression tree analysis of the brain lesion pattern.

Results: Subjects were assigned to olfactory diagnoses of anosmia, hyposmia or normosmia. These diagnoses were predictable at an accuracy of $62.3 \%$ from the degree of damage in the olfactory bulb and in the left temporal lobe pole. The main diagnosis algorithm addressed the presence of anosmia, which could be predicted from the degree of damage in these brain areas at an accuracy of $81.3 \%$.

Conclusions: We independently reproduced previously identified brain regions in which morphological damage is associated with olfactory loss. Based on this reproduction, an algorithm was developed for the diagnosis of anosmia from central-nervous damage. Thus, we introduce a morphological component to the olfactory diagnosis that specifically addresses clinical cases of olfactory loss following head trauma.

Key words: smell, anosmia, head trauma, MRI scan

\section{Introduction}

Brain regions activated by olfactory stimuli in humans ${ }^{(1,2)}$ demonstrate remarkable morphological dynamics ${ }^{(1)}$. This dynamically changing morphology of the olfactory network is attended by variations in olfactory function. For example, volume loss in the gray matter ${ }^{(3)}$ or the olfactory bulb ${ }^{(4-7)}$ is accompanied by reversible anosmia. Normosmia was restored when the volume of olfactory brain areas such as the olfactory bulb was restored ${ }^{(8,9)}$.
This was supported by the observation of statistically significant differences in the right and left olfactory bulb and tract volumes between anosmic and hyposmic patients and between posttraumatic patients and control subjects ${ }^{\left({ }^{6}\right)}$. Thus, the relationship between morphological signs of posttraumatic brain damage in areas particularly important to the sense of smell can be considered as well established.

This relationship between the morphological dynamics of the 
olfactory brain network and olfactory function suggests that anatomical information may be exploited for the diagnosis of an olfactory dysfunction. However, despite this knowledge, including the identification of relevant areas such as the olfactory bulbs and tracts, standard diagnosis of olfactory function relies on patients' performance in psychophysical olfactory tests without regarding the brain morphology.

The present study aimed at obtaining a brain-morphology based criterion that might help physicians to establish the diagnosis of a patient's olfactory function. As associations between the topography of brain damages and olfactory loss had been explored previously at a descriptive level in detail ${ }^{(6)}$, present assessments did not pursue this path towards further details such as association of particular dimensions of olfactory loss with the brain morphology, but remained at the level of major clinical olfactory diagnoses, in particular of anosmia, and focused on obtaining an algorithm for use in the procedure of olfactory diagnosis.

\section{Materials and methods}

Patients and olfactory testing

The study followed the Declaration of Helsinki and was approved by the Ethics Committee of the Faculty of Medicine of the Technical University of Dresden, Germany (protocol number EK287092010). Informed written consent from each participating subject had been obtained. Sixty-four patients (age 22 - 75 years, $50.1 \pm 13.9$ years; 39 men) were enrolled who had presented themselves at the Smell \& Taste Clinic, Department of ORL, TU Dresden, with the symptom "olfactory loss". Forty-one of these patients (age 29 - 75 years, $53.8 \pm 12.7$ years; 23 men) remembered a head trauma dated back $40 \pm 39$ months, of whom 20 were diagnosed as hyposmic and 21 as functionally anosmic. The other subjects (age 22 - 72 years, $43.4 \pm 13.6$ years; 16 men) severed for potential comparison and had no history of brain injury, however, it is known that minor morphological signs of brain damage are among frequent accidental observations during magnet-resonance imaging (MRI) without that the subject actually remembered the actual cause. Of these subjects, 16 were diagnosed as being normosmic, five as hyposmic and two as anosmic. The inclusion of subjects without brain trauma served to avoid a complete overlap of subjects with brain lesions with subjects with olfactory loss.

Following rhinological examination including nasal endoscopy and a detailed structured history ${ }^{(10)}$ the subjects' olfactory acuity was quantified by means of the clinically established "Sniffin' Sticks" test battery ${ }^{(11,12)}$. This assessed the three main components, namely (i) the perception of odours at low concentrations, which is the odour threshold, (ii) the distinction of different smells, which is the ability of odour discrimination, and

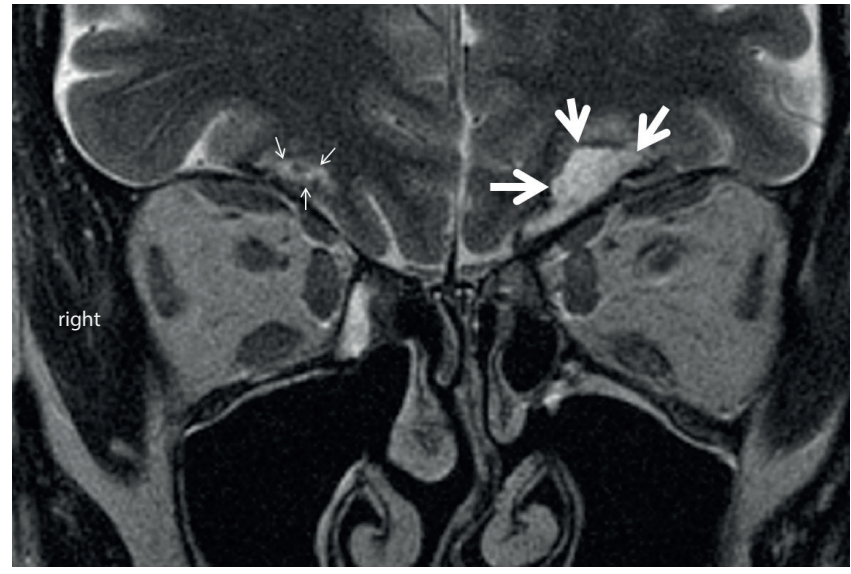

Figure 1. An example MRI scan showing a coronal section through the brain of a patient with 1 st degree lesion at the right OFC and a relatively large 2nd degree lesion with CSF deposit between meninges at the left OFC. The displayed subject was anosmic.

(iii) the ability to name or associate an odour, which is odour identification. The detailed procedure has been described previously ${ }^{(11,12)}$. In brief, olfactory thresholds were obtained for the roselike odour phenylethyl-alcohol at 16 successive 1:2 dilution steps starting at a solution of $4 \%$. Using a three-alternative (one pen with the odorant and two blanks presented at intervals of $3 \mathrm{~s}$ in front of the nostril) forced-choice task and a staircase paradigm, two successive correct or one incorrect identifications triggered a staircase reversal. The odour threshold was the mean of the last four out of seven reversals (normal values $>6^{(13)}$ ). Odour discrimination was determined with triplets of pens, two containing the same odorant and the third a different, "target", odorant, comprising a total of 16 different target/non-target combinations (normal score $\geq 11$ correct discriminations using a three-alternative forced-choice task) (for details, see Table 2 of ${ }^{(14)}$ ). The odour identification subtest comprised 16 commonly known odours ${ }^{(14)}$ and was done using a list of four descriptors from which the subjects had to name the correct odour (normal score: $\geq 12$ correct identifications). The olfactory diagnosis was derived from the "Threshold Discrimination Identification" (TDI) sum score of the subtests ${ }^{(15,16)}$, in which normosmia requires a score $>30.5$ while hyposmia is defined at $30.5 \geq \mathrm{TDI}>15.5$ and functional anosmia at TDI $\leq 15.5)^{(13)}$.

\section{Brain imaging}

Structural MRI was obtained using a Siemens Sonata 1.5 T scanner (Siemens, Erlangen, Germany) using a T1-weighted (turbo flash sequence) axial scan with 192 slices ( $1 \mathrm{~mm}$ ), a $384 * 384$ matrix, flip angle $15^{\circ}$, a repetition time of $2180 \mathrm{~ms}$, echo time of $3.93 \mathrm{~ms}$, and two averages (2180/3.93/2) was acquired (Figure

1). The degree of cerebral lesions was quantified bilaterally (Figure 2) at 11 cerebral areas that have been reported to be involved in the human central nervous processing of smell ${ }^{(1)}$. This included the olfactory bulb, the lateral orbitofrontal cortex, the 



Figure 2. Heat map of brain lesions data.

(columns: brain regions, rows: patients, color code: red = severe damage, yellow = moderate damage, dark gray = not affected), sorted for olfactory diagnoses (left column, anosmia = red, hyposmia = yellow, normal olfactory function $=$ dark gray). Above the heat map, the total number of observed brain lesions per region (column sums of the heat map) is displayed as a bar chart. Abbreviations: olfactory bulb (BO), lateral orbitofrontal cortex (IOFC), median orbitofrontal cortex (mOFC), hypothalamus (hypoth), temporal lobe pole (TLPole), mesial temporal lobe (mesTL), anterior (antins), posterior insular cortex (postlns), thalamus (thal), striatum (stri-at), Globus pallidus (pallid).

median orbitofrontal cortex, the hypothalamus, the temporal lobe pole, the mesial temporal lobe, the anterior and posterior insular cortex, the thalamus, the striatum and the Globus pallidus. The degree of regional brain damage was quantified using a three-point ordinal scale. Absent damage was attributed a value of 0 , moderate damage a value of 1 (lesion clearly present; extent very small) and severe damage a value of 2 (lesion clearly present; large extent). Grading was done by experienced observers (NR, $\mathrm{KH}$ ) and inter-observer incongruences were discussed until a joint decision was reached.

\section{Data analysis}

Analysis focused on establishing a diagnostic algorithm based
Table 1. Detailed explanation of the bilateral olfactory damage score.

\begin{tabular}{|ccc|}
\hline \multicolumn{2}{c}{ Damage score } & BOD \\
\hline One side & Opposite side & \\
\hline 0 & 0 & 0 \\
\hline 0 & 1 & 1 \\
\hline 0 & 2 & 2 \\
\hline 1 & 1 & 4 \\
\hline 1 & 2 & 6 \\
\hline 2 & 2 & 12 \\
\hline
\end{tabular}

The bilateral olfactory damage score is calculated as BOD = $\Sigma$ Damage $_{\text {left,right }} \cdot\left(1+\right.$ minimum(Damage left $_{\text {Damage }}$ right $)$ with Damage scaled as $[0,1,2]$ for increasing severity, i.e., absent damage was attributed a value of 0 , moderate damage a value of 1 and severe damage a value of 2 . The BOD score accommodated the perception that bilateral is functionally more serious that unilateral damage as the function of a damaged brain area may still be provided by its contralateral healthy counterpart. This also agreed with visual inspection of the data that suggested less frequent normosmia in the presence of bilateral brain regional damage. The table demonstrates which possible values can be taken by the BOD score, i.e., in the presence of no (0), moderate (1) or severe (2) damage at one side and the concomitant presence $1-2$ ) or absence $(0)$ of damage at the same brain are of the contralateral side.

on the damage pattern in brain areas that possess a particular importance for olfaction. Visual inspection of the data suggested that normosmia was less frequent in the presence of bilateral brain regional damage (Figure 2). To accommodate this observation, bilateral olfactory damage (BOD) score was developed as BOD $=\Sigma$ Damage $_{\text {left, right }} \cdot\left(1+\right.$ minimum $\left(\right.$ Damage $_{\text {left }}$ Damage $\left._{\text {right }}\right)$, with Damage scaled as $[0,1,2]$ for increasing severity (Table 1 ), separately for each brain area. This agreed with the perception that the function of a damaged brain area may be partly substituted by its intact contralateral equivalent, making bilateral damage functionally more severe than unilateral.

The BOD scores, the original side-specific damage scores, the row sum of the brain damage grades (Figure 2), together with the subjects' age, sex, and the duration of the disorder were submitted to classification and regression tree (CART) analyses (17), from which a diagnostic algorithm was derived. Calculations were done using the library "rpart" (http://cran.r-project.org/ web/packages/rpart/index.html) in R (version 3.0.2 for Linux; http://CRAN.R-project.org/). To estimate the predictive performance of identifying (i) olfactory diagnoses (normosmia, hyposmia, functional anosmia) or, alternatively (ii) just anosmia, i.e., binary as its presence or absence, the tree models were cross- 




Figure 3. Classification and regression tree (CART) visualizing the decision rules for assignment of patients to the three olfactory diagnostic classes, i.e., anosmia (class $=0$, blue), hyposmia (class $=1$, violet) or normosmia (class $=2$, green). Decision steps are colored in orange, results, i.e., diagnoses, are colored blue, violet or green for the three different olfactory diagnoses. CART analysis identified a bilateral olfactory damage score, calculated as:

$\mathrm{BOD}=\Sigma$ Damage $_{\text {left,right }} \cdot\left(1+\operatorname{minimum}\left(\right.\right.$ Damage $_{\text {left }}$ Damage $\left._{\text {right }}\right)$ with Damage (Dmg) scaled as [0,1,2] for increasing severity, at the olfactory bulb scoring at least 2 , a diagnosis of anosmia was most likely while with less pronounced damage, the diagnosis depended on the patient's age, and in subjects aged $<54$ years, normosmia was likely in the absence of lesions in the left temporal lobe pole (TLPole).

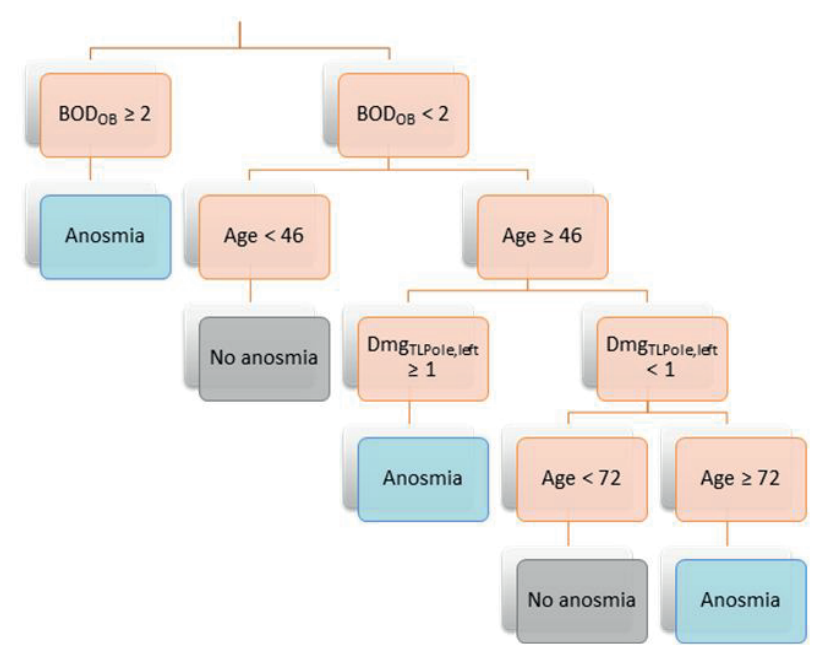

Figure 4. Classification and regression tree (CART) visualizing the decision rules for assignment of subjects to anosmia (class $=1$, blue) or to any other olfactory diagnosis (normal or reduced function: class $=0$, green). Decision steps are colored in orange, results, i.e., the presence or absence of anosmia, are colored blue, or green respectively. CART analysis identified that from bilateral olfactory damage score, calculated as: $\mathrm{BOD}=\Sigma$ Damage $_{\text {left,right }} \cdot\left(1+\right.$ minimum $\left(\right.$ Damage $_{\text {left' }}$ Damage $\left._{\text {right }}\right)$ with Damage (Dmg) scaled as $[0,1,2]$ for increasing severity, at the olfactory bulb from 1.5 upwards, a diagnosis of anosmia was most likely. With less severe damage, an age below 46 years made anosmia unlikely while with older age up to 72 years, anosmia became more likely when the left temporal lobe pole (TLPole) was damaged. validated using a leave-k-out approach, where $\mathrm{k}$ was a randomly picked tenth of the total sample and the tree models were built ten times with the respective remaining data. Finally, the best algorithm, judged by predictivity of the olfactory diagnosis, was assessed using a receiver operating characteristic (ROC) curve analysis with calculation of the area under that curve, using the library "ROCR" in $\mathrm{R}^{(18)}$.

\section{Results}

Olfactory and MRI diagnoses were available from 64 subjects (39 men). Based on their TDI scores, subjects were assigned to diagnoses of functional anosmia ( $n=23,35.9 \%)$, hyposmia $(n=25$, $39.1 \%$ ) or normosmia ( $n=16,25 \%)$. CART analysis associated the olfactory diagnosis not with this sum but with the degree of morphological damage in the olfactory bulb and in the left temporal lobe pole (Figure 3). Specifically, from a BOD score at the olfactory bulb of 2 upwards, a diagnosis of anosmia was most likely. With less pronounced damage of the olfactory bulb, the diagnosis depended firstly on the patient's age, i.e., patients older than 54 years were more likely to have reduced olfactory function (hyposmia). Among younger subjects, the olfactory diagnosis of normosmia depended on the absence of damage in the (left) temporal lobe pole. The CART model provided a cross- validated correct diagnosis in $62.3 \%$ of the cases.

The same anatomical regions were also found when focusing a further CART analysis on the target diagnosis anosmia (Figure 4). Again, from a BOD score at the olfactory bulb of 2 upwards, a diagnosis of anosmia was most likely. With less severe damage, younger subjects aged below 46 years were unlikely to display anosmia while in subjects aged between 46 and 72 years the diagnosis of anosmia depended on the presence of a damage of the temporal lobe pole (BOD score 1 or higher). The cross-validated correct diagnosis of anosmia using the CART tree was 81.3 $\%$. This algorithm resulted in an area under the ROC curve of 0.8 , indicating a good diagnostic criterion according to the traditional interpretation of the curve, and a specificity and sensitivity of 0.74 and 0.85 , respectively.

\section{Discussion}

The addition of a morphological criterion to the psychophysical test results meets a desire in olfactory diagnostics. It may strengthen its acceptance in counseling cases where olfactory loss following brain injury may be the cause of compensation claims against insurances or culpable party. The CART decision rules did not provide complete (100 \%) correct diagnosis when prospectively cross-validated. For the diagnosis of anosmia, which 
is often the main aim in olfactory counseling in legal context, an accurate prediction was possible in $80 \%$ of the cases, as also emphasized by the ROC area agreeing with the interpretation of a good yet not excellent diagnostic criterion. The morphological component may reasonably complement the functional tests in olfactory diagnosis but it will not replace them. This follows from the fact that brain damage after trauma accounts for only $5 \%$ of all olfactory losses ${ }^{(19)}$. However, olfactory loss is frequent after brain injury ${ }^{(20)}$ and moreover, brain damage may be found without that the subject can recall an incident of injury. A contribution of a rupture of the olfactory nerves to the olfactory loss ${ }^{(21)}$ cannot be excluded in the present data as morphological information about the olfactory nerve was not accessible; however, by having identified a role of particular brain regions for olfactory loss rather than the mere cumulative sum of brain damage across the analysed regions, the present results support that central-nervous damage con-tributes to the degree and recovery rate of posttraumatic olfactory loss ${ }^{(6)}$. The results also agree with previous evidence that olfactory function depended on lesions in two brain regions, comprising the olfactory bulb and olfactory tract ${ }^{(20)}$, in particular the temporal lobe pole ${ }^{(22)}$. The present results compiled from this damage a morphologybased diagnostic algorithm, particularly well performing for anosmia, which is the major addition of this analysis to previous knowledge. While the highly significant role of the olfactory bulb is very clear, less is known about a possible role of the temporal lobe pole in olfaction. Lesions induced by radiation have been found to be associated with decreased olfactory function ${ }^{(23)}$. In addition, specific deficits of the temporal lobe pole in primary progressive aphasia have been found to be related severe deficits in odour naming and matching. The role of the temporal lobe pole was seen as "linking odour object representations to transmodal networks, given its anatomical proximity to olfactory and visual object processing areas" (24). Similarly, patients with fronto-temporal lobar degeneration had significant problems to identify retronasally presented odours ${ }^{(25)}$. These results are further supported by other studies emphasizing the important role of the temporal lobe pole in the human processing of odours ${ }^{(26,27)}$. Interestingly, a side specificity of the left temporal pole was observed, possibly pointing at a greater importance of the left side for olfactory function than of the right side. However, there is a growing body of research indicating that there is no clear laterality in the olfactory system (for reviews see ${ }^{(28,29)}$ ). Accordingly, more research is needed to confirm the present findings on the possible lateralized functioning of the temporal lobe within the olfactory system.

The inclusion of age in both CARTs is not surprising. Higher age was associated with a higher probability of olfactory loss. This is consistent with the well-known decline in human olfactory function with increasing age ${ }^{(20,31)}$. A further factor could have been sex, for which a disadvantage of the male sex with respect to olfactory performance has been repeatedly reported ${ }^{(32)}$. However, in the present cohort sex played no role for the olfactory function. It was neither included in the CART, which might be a consequence of the lack of significant sex differences when analysed separately (Pearson $\chi^{2}: 0.67, p=0.72$ ).

An accuracy of $80 \%$ in identifying olfactory loss leaves room for improvement. Nevertheless, with a ROC area of 0.8 (with an ideal maximum of 1) the algorithm reaches already the limit of a test usually considered as "good". With respect to the sensitivity and specificity, it is not far below that of olfactory screening tests. For example, the Connecticut Smell Test (CST) derived from the comprehensive based on the Connecticut Chemosensory Clinical Research Center (CCCRC) test ${ }^{(33)}$ was initially reported with a sensitivity and a specificity to detect olfactory loss of 88 and $77 \%$, respectively, ${ }^{(34)}$ and in a subsequent evaluation with a sensitivity and a specificity of 93.3 and $76 \%{ }^{(35)}$. The present algorithm may provide support for the functional diagnosis by a second and independent morphological criterion. This may strengthen the case for example in medico-legal settings. The test could only include more frequent brain lesions as several of the candidate regions could not be analysed as lesions were to rate in the present cohort. When applying the present analytical approach to a larger data set and including subjects with normosmia but brain damages, who unfortunately were not available for enrollment in the present study, an improvement of the morphological criterion may be expected with good reason.

\section{Conclusions}

In conclusion, we independently identified the same brain regions, in which morphological damage is strongly associated with olfactory loss, that had been shown 15 years ago ${ }^{(6)}$. We came to these conclusions by using alternative bioinformatics. Based on this reproduction, we used the thus valid morphological criteria and developed an algorithm for the diagnosis of anosmia from central-nervous damage. This adds to other causes such as olfactory nerve rupture not addressed here. Thus, we provide a brain morphological component to the olfactory diagnosis that specifically addresses clinical cases of olfactory loss following head trauma.

\section{Acknowledgement}

The work has been partly supported by the Landesoffensive zur Entwicklung wissenschaftlich-ökonomischer Exzellenz (LOEWE, JL), Schwerpunkt: Anwendungsorientierte Arzneimittelforschung. The funders had no role in method design, data selection and analysis, decision to publish, or preparation of the manuscript. We would like to thank Maren Eckhardt for her help with some of the analyses.

\section{Author contributions}

Conceived and designed the experiments: TH, KH. Performed 


\section{References}

1. Gottfried JA. Smell: central nervous processing. Adv Otorhinolaryngol. 2006; 63: 44-69.

2. Savic I. Imaging of brain activation by odorants in humans. Curr Opin Neurobiol. 2002; 12: 455-461.

3. Bitter T, Gudziol H, Burmeister HP, Mentzel H-J, Guntinas-Lichius O, Gaser C. Anosmia leads to a loss of gray matter in cortical brain areas. Chem Senses. 2010; 35: 407415.

4. Meisami E. Effects of olfactory deprivation on postnatal growth of the rat olfactory bulb utilizing a new method for production of neonatal unilateral anosmia. Brain Res. 1976; 107: 437-444

5. Rombaux P, Duprez T, Hummel T. Olfactory bulb volume in the clinical assessment of olfactory dysfunction. Rhinology. 2009; 47: 3-9.

6. Yousem DM, Geckle RJ, Bilker WB, Kroger H, Doty RL. Posttraumatic smell loss: relationship of psychophysical tests and volumes of the olfactory bulbs and tracts and the temporal lobes. Academic Radiol. 1999; 6: 264-272.

7. Mueller A, Abolmaali ND, Hakimi AR, et al. Olfactory bulb volumes in patients with Idiopathic Parkinson's Disease. J Neural Transm. 2005; 112: 1363-1370

8. Gudziol V, Buschhuter D, Abolmaali N Gerber J, Rombaux P, Hummel T. Increasing olfactory bulb volume due to treatment of chronic rhinosinusitis--a longitudinal study. Brain. 2009; 132: 3096-3101

9. Royet JP, Plailly J, Saive AL, Veyrac A, DelonMartin C. The impact of expertise in olfaction. Front Psychol. 2013; 4: 928.

10. Welge-Luessen A, Leopold DA, Miwa T. Smell and Taste Disorders - Diagnostic and Clinical Work-Up. In: Welge-Luessen A, Hummel T, editors. Management of Smell and Taste Disorders - A Practical Guide for Clinicians. Stuttgart: Thieme; 2013. p. 49-57.

11. Hummel T, Sekinger B, Wolf SR, Pauli E, Kobal G. 'Sniffin' Sticks': Olfactory performance assessed by the combined testing of odor identification, odor discrimination and olfactory threshold. Chem Senses. 1997; 22: 39-52.

12. Kobal G, Hummel T, Sekinger B, Barz S, Roscher S, Wolf SR. "Sniffin' Sticks": Screening of olfactory performance. Rhinology. 1996; 34: 222-226.

13. Hummel T, Kobal G, Gudziol H, Mackay-Sim A. Normative data for the "Sniffin' Sticks" including tests of odor identification, odor discrimination, and olfactory thresholds: an upgrade based on a group of more than 3,000 subjects. Eur Arch Otorhinolaryngol. 2007; 264: 237-243.

14. Hummel T, Sekinger B, Wolf S, Pauli E, Kobal G. "Sniffin' Sticks": Olfactory performance assessed by the combined testing of odor identification, odor discrimination and olfactory threshold. Chem Senses. 1997; 22: 39-52.

15. Kobal G, Klimek $L$, Wolfensberger $M$, et al. Multicenter investigation of 1,036 subjects using a standardized method for the assessment of olfactory function combining tests of odor identification, odor discrimination, and olfactory thresholds. Eur Arch Otorhinolaryngol. 2000; 257: 205-211.

16. Wolfensberger M, Schnieper I, WelgeLussen A. Sniffin'Sticks: a new olfactory test battery. Acta Otolaryngol. 2000; 120: 303306.

17. Breimann L, Friedman JH, Olshen RA, Stone CJ. Classification and Regression Trees. Boca Raton: Chapman and Hall; 1993.

18. Sing T, Sander O, Beerenwinkel N, Lengauer T. ROCR: visualizing classifier performance in R. Bioinformatics (Oxford, England). 2005; 21: 3940-3941.

19. Damm M, Temmel A, Welge-Lüssen A, et al. Epidemiologie und Therapie von Riechstörungen in Deutschland, Österreich und der Schweiz. HNO. 2004; 52: 112-120.

20. Sumner D. Post-traumatic anosmia. Brain. 1964; 87: 107-120.

21. Delank KW, Fechner G. [Pathophysiology of post-traumatic anosmia]. Laryngorhinootologie. 1996; 75: 154-159.

22. Gentry LR, Godersky JC, Thompson B. MR imaging of head trauma: review of the distribution and radiopathologic features of traumatic lesions. AJR Am J Roentgenol. 1988; 150: 663-672.

23. Leyrer CM, Chan MD, Peiffer AM, et al. Taste and smell disturbances after brain irradiation: a dose-volume histogram analysis of a prospective observational study. Pract Radiat Oncol. 2014; 4: 130-135.

24. Olofsson JK, Rogalski E, Harrison T, Mesulam MM, Gottfried JA. A cortical pathway to olfactory naming: evidence from primary progressive aphasia. Brain. 2013; 136: 12451259.

25. Omar R, Mahoney CJ, Buckley AH, Warren JD. Flavour identification in frontotemporal lobar degeneration. J Neurol Neurosurg Psychiatry. 2013; 84: 88-93.

26. Olson IR, Plotzker A, Ezzyat Y. The Enigmatic temporal pole: a review of findings on social and emotional processing. Brain.
2007; 130: 1718-1731.

27. Kasai K, Shenton ME, Salisbury DF, et al. Differences and similarities in insular and temporal pole MRI gray matter volume abnormalities in first-episode schizophrenia and affective psychosis. Arch Gen Psychiatry. 2003; 60: 1069-1077.

28. Doty RL, Bromley SM, Moberg PJ, Hummel T. Laterality in human nasal chemoreception. In: Christman S, editor. Cerebral asymmetries in sensory and perceptual processing. Amsterdam: North Holland Publishing; 1997. p. 497-542.

29. Seubert J, Freiherr J, Djordjevic J, Lundstrom JN. Statistical localization of human olfactory cortex. Neuroimage. 2012; 66c: 333-342.

30. Venstrom D, Amoore JE. Olfactory threshold in relation to age, sex, or smoking. J Food Sci. 1968; 33: 264-265.

31. Doty RL, Shaman P, Applebaum SL, Giberson R, Sikorski L, Rosenberg L. Smell identification ability: changes with age. Science. 1984; 226: 1441-1443.

32. Doty RL. Gender and endocrine-related influences on human olfactory perception. In: Meiselman R, editor. Clinical Measurement of Taste and Smell. New York: MacMillan; 1986. p. 377-413.

33. Cain WS. Testing olfaction in a clinical setting. EarNoseThroatJ. 1989; 68: 316: 22-28.

34. Toledano A, González E, Rodríguez G, Galindo AN. [Development of an olfactory screening test based on the Connecticut test (CCCRC)]. Acta Otorrinolaringol Esp. 2005; 56: 116-121.

35. Toledano A, Ruiz C, Navas C, et al. Development of a short olfactory test based on the Connecticut Test (CCCRC). Rhinology. 2009; 47: 465-469.

\author{
Jörn Lötsch \\ Institute of Clinical Pharmacology, \\ Goethe - University \\ Theodor-Stern-Kai 7 \\ 60590 Frankfurt am Main \\ Germany \\ Phone: +49-69-6301-4589 \\ Fax: +49-69-6301-4354 \\ E-mail: j.loetsch@em.uni-frankfurt.de
}

